\title{
Anorectal Manometry Versus Patient-Reported Outcome Measures as a Predictor of Maximal Treatment for Fecal Incontinence
}

\author{
Lisa Ramage ${ }^{1}$, Shengyang Qiu ${ }^{1}$, Zhu Yeap $^{1}$, Constantinos Simillis ${ }^{1}$, Christos Kontovounisios ${ }^{1,2}$, \\ Paris Tekkis ${ }^{1,2}$, Emile Tan ${ }^{3}$ \\ ${ }^{1}$ Department of Surgery and Cancer, Chelsea and Westminster Hospital, Imperial College London NHS Trust, London; ${ }^{2}$ Department of \\ Colorectal Surgery, The Royal Marsden NHS Foundation Trust, London, UK; ${ }^{3}$ Singapore General Hospital, Singapore
}

Purpose: This study aims to establish the ability of patient-reported outcome measures (PROMs) and anorectal manometry (ARM) in predicting the need for surgery in patients with fecal incontinence (FI).

Methods: Between 2008 and 2015, PROMs data, including the Birmingham Bowel and Urinary Symptoms Questionnaire (BBUSQ), Short Form 36 (SF-36), Wexner Incontinence Score and ARM results, were prospectively collected from 276 patients presenting with FI. Spearman rank was used to assess correlations between specific PROMs questions and ARM assessments of sphincter motor function. Binomial regression analyses were performed to identify factors predictive of the need for surgery. Finally, receiver operating characteristic (ROC) curve analyses were performed to establish the utility of individual ARM and PROMs variables in predicting the need for surgical intervention in patients with FI.

Results: Two hundred twenty-eight patients $(82.60 \%)$ were treated conservatively while $48(17.39 \%)$ underwent surgery. On univariate analyses, all 4 domains of the BBUSQ, all 8 domains of the SF-36, and the Wexner Incontinence Score were significant predictors of surgery. Additionally, maximum resting pressure, 5-second squeeze endurance, threshold volume, and urge volume were significant. On ROC curve analyses, the only significant ARM measurement was the 5-second squeeze endurance. PROMs, such as the incontinence domain of the BBUSQ and five of the SF-36 domains, were identified as fair discriminators of the need for surgery.

Conclusion: PROMs are reliable predictors of maximal treatment in patients with FI and can be readily used in primary care to aid surgical referrals and can be applied in hospital settings as an aid to guide surgical treatment decisions.

Keywords: Anorectal physiology; Anorectal manometry; Patient-reported outcome measures; Fecal incontinence

\section{INTRODUCTION}

Fecal incontinence (FI) is defined as the involuntary loss of liquid and/or solid stool through the anus [1]. Its prevalence is likely un-

Received: October 26, 2017 - Accepted: October 16, 2018

Correspondence to: Christos Kontovounisios, M.D. PhD. FACS. FRCS.

Department of Surgery and Cancer, Chelsea and Westminster Hospital, 369 Fulham Road, London, SW10 9NH, UK

Tel: +447845589286, Fax: +447845589286,

E-mail: c.kontovounisios@imperial.ac.uk

ORCID code: https://orcid.org/0000-0002-1828-1395

(C) 2019 The Korean Society of Coloproctology

This is an open-access article distributed under the terms of the Creative Commons Attribution NonCommercial License (http://creativecommons.org/licenses/by-nc/4.0) which permits unrestricted noncommercial use, distribution, and reproduction in any medium, provided the original work is properly cited. derestimated due to underreporting by its sufferers, but is quoted to be in the region of $7.7 \%$ (range, $2.0 \%-20.7 \%$ ) [2]. The presence of FI has a major negative impact on physical and psychological health and on lifestyle; in many cases, it can lead to severe social restrictions. Treatment ranges from conservative treatments such as lifestyle adaptations, stool-bulking agents, biofeedback, sphincter exercises, and transanal irrigation to more invasive procedures such as sacral nerve stimulation (SNS) and sphincter repair [3].

Anorectal manometry (ARM) has long been used as part of the diagnostic work-up in the assessment of patients with FI. Several parameters, including the resting pressures (RPs) of the anal canal, maximal squeeze pressure increment (MSPI), squeeze endurance (SE), and measurements of rectal sensitivity, are routinely assessed in patients with FI who are referred to pelvic-floor special- 
ists. Nevertheless, the diagnostic accuracy of ARM for patients with FI remains unclear. The sensitivity and the specificity in diagnosing FI varies significantly between studies, and whether any correlation exists between ARM measurements and the severity of symptoms is unclear. Overall, how ARM influences clinical decision making as to the most appropriate treatment for patients is still unclear.

For the above reasons, this study aimed to assess the ability of ARM, compared to patient-reported outcomes, in predicting the need for surgical intervention in patients suffering with FI. Additionally, this study also aimed to ascertain the degree of correlation between patient-reported outcome measures (PROMs) and ARM measurements specific to external and internal anal sphincter function.

\section{METHODS}

Data were prospectively collected between 2008 and 2015 from adult patients undergoing assessment of and treatment for FI at a single center specializing in the management of pelvic-floor disorders. Ethical approval was granted by the Bristol NRES Committee (14/SW/0051) for the maintenance of a research database with prospective patient consent for data inclusion. Prior to this data was collated as part of ongoing clinical care and to assess response to treatment and stored anonymously for audit purposes. At the first consultation, patients underwent clinical assessments, including the use of several validated PROMs questionnaires, in addition to physical examinations and ARM measurements. Additionally, patients underwent endoanal sphincter ultrasound assessments to determine whether any anal sphincter defects were present.

All patients diagnosed with FI were initially referred for pelvicfloor physiotherapy (biofeedback) and/or started on stool-bulking agents (e.g., loperamide). Patients were followed up at 6- to 12-month intervals. Patients were discharged if FI symptoms were sufficiently managed. Patient who had significant and persistent symptoms were considered for either SNS or surgical sphincter repair as per National Institute of Clinical Excellence guidelines for the management of FI in adults [3].

Patients were included in this study if they had recorded data within the database from an initial consultation within the pelvicfloor clinic and prior to any interventions taking place. The exception to this was patients who had sustained an obstetric anal sphincter injury (OASI); invariably, such patients had received some general pelvic-floor physiotherapy prior to consultation with a pelvic-floor colorectal surgeon. Patients had to have undergone an ARM assessment of sphincter function, with completion of at least one PROM questionnaire (WIS, BBUSQ, and SF-36).

The primary outcome measure was whether patients required only conservative treatment or additional surgery (in the form of either SNS or sphincter repair). The secondary outcome measure was the degree of correlation between commonly used ARM pa- rameters, which are considered as markers of internal or external sphincter motor function, and specific patient symptoms relating to abnormalities of internal and external sphincter function. The latter was assessed in order to determine whether specific question stems detailed within the BBUSQ had the potential to act as surrogate markers in the community for the likelihood of achieving poor ARM results.

\section{Patient-reported outcome measures}

PROM measures were collected for research purposes only and were not readily available to the clinical team; therefore, individual questionnaire responses were not used in clinical decisionmaking. Rather, decisions regarding appropriate steps of care were based upon both a clinical review of symptoms, both subjectively and objectively, and a review of ARM, Endoanal ultrasound and other imaging results. Patients were asked to complete 2 clinically validated symptoms questionnaires, the Birmingham Bowel and Urinary Symptoms Questionnaire (BBUSQ) [4] and Wexner Incontinence Score (WIS) [5], prior to the initial clinical assessment. The BBUSQ score evaluated the severity of pelvic-floor symptoms through the assessments of four major symptom domains (constipation, FI, fecal evacuatory disorder, and urinary symptoms). Scores for each of the four domains were quantified as scores out of 100 , with a higher score indicating worse symptom severity. The WIS, marked out of 20, quantified the severity of FI, with a higher score indicating worse symptoms.

The Short Form 36 (SF-36) quality of life (QoL) score was also collected [6]. This has 8 scoring domains, including vitality, physical functioning, bodily pain, general health perceptions, physical role functioning, emotional role functioning, social role functioning, and mental health. Each domain is scored out of 100, with a higher score indicating a better QoL. Functional and QoL data collected as detailed above were anonymized and entered into the prospectively maintained pelvic-floor research database.

\section{Anorectal manometry}

After the patients had given informed consent, they were positioned in the left lateral position. ARM was carried out using the T-DOC Air-Charged ARM Catheters (LABORIE International, Mississauga, ON, Canada), which have four pressure sensors measuring 4 quadrants of the anal sphincter, plus a distal balloon that can be filled to obtain sensory responses from the patients. The pressure sensors were connected to the Delphis IP processor (LABORIE International), which served to pump air to prime the catheters and to digitize and record pressure readings from the pressure sensors. Digital rectal examination was performed prior to introduction of the ARM catheter into the anal canal.

The maximal RP was recorded at the level of the high-pressure zone. The patients were then asked to squeeze their anal sphincter as hard as they could in order to determine the MSPI and then to squeeze and hold for 5 seconds to determine the SE. A mean of three readings was taken for each of the motor function parame- 
ters. Rectal sensation was measured by inflating a balloon on the catheter's tip with air while it was being placed within the rectum. The threshold volume (TV), the volume to defecatory urge, and the maximal tolerated volumes were measured. The rectal anal inhibitory reflex was assessed by inflating the intrarectal balloon with $50 \mathrm{~mL}$ of air. Although newer ARM modalities became available during the study period, all patients in this study underwent manometry using the calibrated air-filled system to ensure consistency throughout the study group.

\section{Statistical methods}

Demographic data from patients who required surgical treatment were compared with the corresponding data from those who did not by using the chi-square test for categorical data, the unpaired $\mathrm{t}$-test for parametric variables and the Whitney-Man U-test for nonparametric variables. Binary logistical regression analyses were performed for ARM, BBUSQ, and SF-36 scores to determine significant variables for determining the maximal treatment required. A P-value of $<0.05$ was considered to be statistically significant.

The predictive abilities of ARM measurements, BBSUQ domains, WIS, and SF-36 domains were assessed by constructing receiver operating characteristic (ROC) curves, with the outcome measure being whether a patient required surgical treatment for FI (SNS and other surgery). The optimum cutoff for each variable, and its respective sensitivity and specificity were determined by calculating the Youden Index for each ROC curve [7]. ROC curves for which the area under the curves (AUCs) were greater than 0.7 were deemed to be fair discriminators of the need for maximal treatment in patients with FI.

\section{RESULTS}

Between 2008-2015, 432 patients were assessed with FI. Of those, 156 patients were excluded due to incomplete data. Therefore, a total of 276 patients were included in this analysis. The patient population was predominantly female ( 248 females vs. 28 males). The mean overall age of the group was 43.59 years. Sphincter weakness was the major etiology (79.3\% of all patients), predominantly secondary to prior obstetric trauma. Two hundred twentyeight patients $(82.6 \%)$ received only conservative treatment while 48 (17.4\%) underwent surgical intervention, either in the form of SNS or sphincter reconstructive surgery (Table 1). Sixty patients $(21.7 \%)$ received no further treatment after initial consultation with the colorectal pelvic-floor team; the vast majority of those were patients who were assessed following OASI and had received some form of generalized pelvic-floor physiotherapy during their

Table 1. Numbers of patients undergoing conservative and surgical management

\begin{tabular}{lc}
\hline Treatment & No. of patients \\
\hline Conservative & 60 \\
None & 152 \\
Biofeedback & 16 \\
Transanal irrigation & \\
Surgical & 37 \\
Sacral nerve stimulation & 11 \\
Other surgery & 276 \\
\hline Total &
\end{tabular}

Table 2. Patients' demographics

\begin{tabular}{|c|c|c|c|c|}
\hline \multirow{2}{*}{ Variable } & \multicolumn{3}{|c|}{ Maximal treatment } & \multirow{2}{*}{ P-value } \\
\hline & Conservative $(n=228)$ & Surgical $(n=48)$ & All/total $(n=276)$ & \\
\hline Sex, female : male & $211: 17$ & $37: 11$ & $248: 28$ & $0.001^{\star, a}$ \\
\hline Age (yr) & $41.25 \pm 14.30$ & $54.63 \pm 15.11$ & $43.59 \pm 15.28$ & $0.000^{*, b}$ \\
\hline \multicolumn{5}{|l|}{ Etiology } \\
\hline \multicolumn{5}{|l|}{ Sphincter weakness } \\
\hline Postsurgical sphincter injury & $7(3.07)$ & $3(6.25)$ & $10(3.62)$ & $0.386^{*}$ \\
\hline Obstetric sphincter injury & $170(74.56)$ & 23(47.92) & $193(69.93)$ & $0.001^{\star, a}$ \\
\hline Sphincter defect unknown etiology & $1(0.44)$ & $1(2.08)$ & $2(0.72)$ & $0.318^{*}$ \\
\hline No sphincter defect & $11(4.82)$ & $2(4.17)$ & $13(4.71)$ & $1.000^{*}$ \\
\hline Neuropathic/CNS & $8(3.51)$ & $1(2.08)$ & $9(3.26)$ & $1.000^{*}$ \\
\hline Proctitis & $2(0.88)$ & $1(2.08)$ & $3(1.09)$ & $0.438^{*}$ \\
\hline Structural pelvic floor abnormality (e.g., intussusception) & $9(3.95)$ & $1(2.08)$ & $10(3.62)$ & $1.000^{*}$ \\
\hline Prior rectal resection (e.g., anterior resection) & $5(2.19)$ & $10(20.8)$ & $15(5.43)$ & $0.000^{*}, \mathrm{a}$ \\
\hline Paradoxical puborectalis contraction & $2(0.88)$ & $0(0)$ & $2(0.72)$ & $1.000^{*}$ \\
\hline Idiopathic & $13(5.70)$ & $6(12.5)$ & 19 (6.88) & $0.099^{*}, \mathrm{a}$ \\
\hline
\end{tabular}

Values are presented as mean \pm standard deviation or number (\%).

CNS, central nervous system.




recovery period. Increasing age and male gender were found to be significant risk factors for undergoing surgical intervention. In terms of etiologies, significant differences were found in the proportions of patients with OASI and those who had undergone rectal resection between the conservative-treatment and the surgical groups (Table 2).

The RP was significantly lower in the surgery group $(\mathrm{P}=0.001)$; however, no statistically significant differences in the other anorectal physiology parameters were found between the groups (Table 3). Age, all domains of the BBSUQ and the SF-36, and the WIS had significant differences in the mean scores between the conservative-treatment and the surgical groups.

\section{Logistic regression analysis}

Binomial logistic regression analyses were performed using continuous data for each of the variables (Table 4 ).

Of the ARP results, maximum $\mathrm{RP}(\mathrm{P}=0.001)$, 5-second squeeze increment $(\mathrm{P}=0.017)$, TV to distension $(\mathrm{P}=0.043)$, and urge volume to distension $(\mathrm{P}=0.033)$ were found to be statistically significant. Of the remaining parameters, age, sex, the WIS, and all domains of the BBSUQ and SF36 were found to have regression significance.

\section{Receiver-operator curve analysis}

ROC curve analyses were performed on the variables to determine their abilities to predict maximal treatment. Variables with an AUC of greater than 0.7 were interpreted as being fair predictors of the need for surgical intervention (Table 5). On ROC curve analyses, the only ARM parameter that had an AUC greater than 0.7 was the 5 -second squeeze increment. Patient age, the incontinence domain of the BBUSQ, and 5 domains of the SF-36 (physical function, physical role, mental health, social function, and general health) were shown to have AUCs of greater than 0.7. The evacuatory domain of the BBUSQ and the WIS had AUCs that were just slightly less than 0.7 .

The Youden index was then used to calculate the optimum cut-

Table 3. Means and standard deviations of patients' variables for different treatment groups

\begin{tabular}{|c|c|c|c|}
\hline Variable & Conservative & Surgical & P-value ${ }^{a}$ \\
\hline Age (yr) & $41.25 \pm 14.3$ & $54.63 \pm 15.1$ & $0.000^{*}$ \\
\hline \multicolumn{4}{|l|}{ Anorectal manometry } \\
\hline Anal canal length $(\mathrm{cm})$ & $2.94 \pm 0.4$ & $3.01 \pm 0.2$ & 0.170 \\
\hline Maximum resting pressure (mmHg) & $61.68 \pm 18.6$ & $51.98 \pm 17.2$ & $0.001^{*}$ \\
\hline Maximum squeeze increment (mmHg) & $35.21 \pm 29.8$ & $29.87 \pm 22.2$ & 0.245 \\
\hline 5-sec squeeze increment (mmHg) & $27.59 \pm 19.1$ & $21.46 \pm 6.8$ & 0.054 \\
\hline Involuntary cough pressure (mmHg) & $46.17 \pm 23.9$ & $44.10 \pm 15.5$ & 0.623 \\
\hline Threshold volume to distension (mL) & $31.18 \pm 12.6$ & $36.42 \pm 23.3$ & 0.217 \\
\hline Urge volume to distension (mL) & $56.49 \pm 18.8$ & $63.50 \pm 25.1$ & 0.119 \\
\hline Maximum tolerated volume (mL) & $100.56 \pm 28.7$ & $104.48 \pm 35.4$ & 0.962 \\
\hline \multicolumn{4}{|l|}{ BBUSQ } \\
\hline Constipation & $54.07 \pm 36.9$ & $44.90 \pm 20.3$ & $0.019^{*}$ \\
\hline Incontinence & $29.18 \pm 17.7$ & $46.06 \pm 25.0$ & $0.000^{*}$ \\
\hline Evacuatory & $13.89 \pm 11.5$ & $20.73 \pm 11.6$ & $0.000^{*}$ \\
\hline Urinary & $19.68 \pm 12.8$ & $27.18 \pm 19.0$ & $0.028^{*}$ \\
\hline WIS & $6.24 \pm 3.4$ & $8.96 \pm 4.7$ & $0.000^{\star}$ \\
\hline \multicolumn{4}{|l|}{ SF-36 } \\
\hline Physical function & $83.30 \pm 22.2$ & $63.77 \pm 31.7$ & $0.000^{*}$ \\
\hline Role physical & $78.62 \pm 24.2$ & $58.33 \pm 32.6$ & $0.000^{*}$ \\
\hline Role emotional & $86.35 \pm 23.16$ & $69.10 \pm 32.7$ & $0.000^{*}$ \\
\hline Vitality & $54.07 \pm 17.2$ & $45.40 \pm 21.5$ & $0.001^{*}$ \\
\hline Mental health & $73.79 \pm 15.8$ & $59.26 \pm 21.6$ & $0.000^{*}$ \\
\hline Social function & $76.40 \pm 21.9$ & $51.30 \pm 27.4$ & $0.000^{*}$ \\
\hline Pain & $75.08 \pm 22.7$ & $63.28 \pm 29.1$ & $0.018^{*}$ \\
\hline General health & $68.33 \pm 18.1$ & $55.08 \pm 20.8$ & $0.000^{*}$ \\
\hline
\end{tabular}

BBUSQ, Birmingham Bowel and Urinary Symptoms Questionnaire; WIS, Wexner Incontinence Score; SF-36, Short Form 36, quality of life questionnaire. ${ }^{*} \mathrm{P}<0.05$. ${ }^{a}$ Mann-Whitney U-test. 
Table 4. Binomial logistic regression analysis of patients' variables

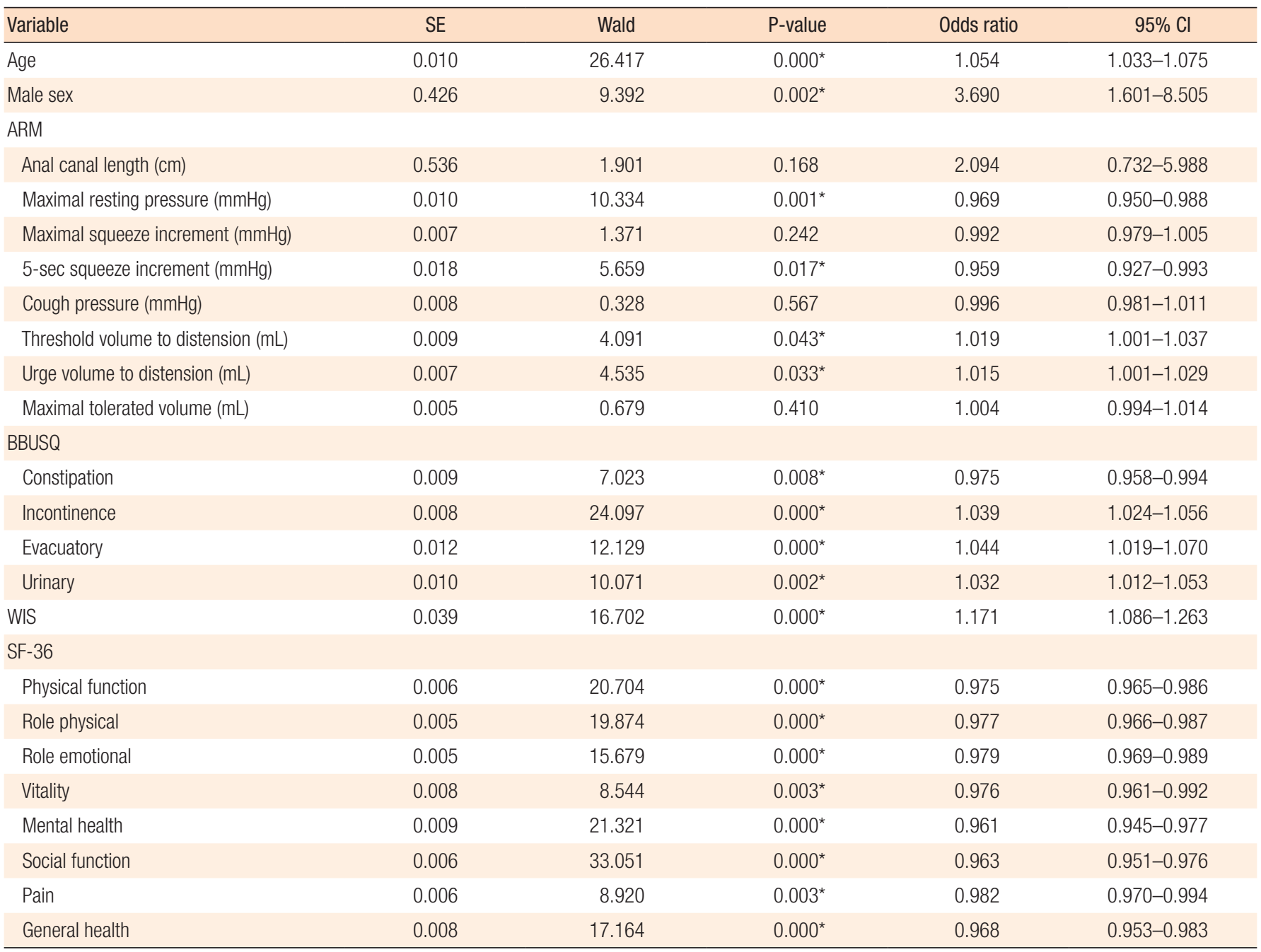

SE, standard error; Cl, confidence interval; ARM, anorectal manometry; BBUSQ, Birmingham Bowel and Urinary Symptoms Questionnaire; WIS, Wexner Incontinence Score; SF-36, Short Form 36, quality of life questionnaire.

${ }^{\star} \mathrm{P}<0.05$.

off points for each of the variables to obtain the highest sensitivities and specificities [7]. A 5-second squeeze increment of less than $16.5 \mathrm{mmHg}$ had a sensitivity of $81.8 \%$ and a specificity of $70.3 \%$ in detecting the need for surgical intervention while a SF36 social function domain score of less than $68.75 / 100$ had a sensitivity of $80 \%$ and a specificity of $69.7 \%$. A BBUSQ incontinence domain score of 39.58 or above had a sensitivity of $65.1 \%$ and specificity of $70.1 \%$ in the detection of the need for surgical treatment. AUC plots for age, 5-second squeeze increment, BBUSQ Incontinence domain, and SF-36 social function domain are shown in Fig. 1.

\section{DISCUSSION}

This is, to our knowledge, the first study to attempt to use ARM results to predict the need for surgical intervention in patients with FI. Within our series, on the univariate binomial regression analyses, a number of factors were statistically significant in the prediction of the need for surgery in FI patients. With regards to sphincter motor function, the resting pressure and the 5-second squeeze increment were significant. On sensory-function testing, threshold and urge volumes were also significant. All parameters of different PROMs were significant. When ROC analyses were performed, the majority of ARM measurements taken had a low sensitivity and specificity in the prediction of the need for sphincter repair or SNS in patients with FI. The only factor that appeared to be indicative of the need for intervention was a poor endurance squeeze pressure (SP) increment.

Few studies in the literature report on attempts to use ARM in the prediction of maximal treatment for patients with FI. Hill et 
Table 5. ROC curve analyses of patients' variables

\begin{tabular}{|c|c|c|c|c|c|c|c|}
\hline Variable & AUC & SE & P-value & $95 \% \mathrm{Cl}$ & Cutoff [7] & Sensitivity & Specificity \\
\hline Age & $0.748^{\mathrm{a}}$ & 0.039 & 0.000 & $0.672-0.823$ & 37.61 & 0.854 & 0.573 \\
\hline \multicolumn{8}{|l|}{ ARM } \\
\hline Anal canal length (cm) & 0.539 & 0.042 & 0.391 & $0.457-0.622$ & 2.75 & 0.979 & 0.127 \\
\hline Maximal resting pressure $(\mathrm{mmHg})$ & 0.648 & 0.043 & 0.001 & $0.563-0.732$ & 56.00 & 0.667 & 0.583 \\
\hline Maximal squeeze increment (mmHg) & 0.556 & 0.046 & 0.225 & $0.465-0.647$ & 27.50 & 0.604 & 0.531 \\
\hline 5-sec squeeze increment (mmHg) & $0.762^{\mathrm{a}}$ & 0.066 & 0.005 & $0.633-0.891$ & 16.50 & 0.818 & 0.703 \\
\hline Involuntary cough pressure (mmHg) & 0.555 & 0.115 & 0.606 & $0.330-0.780$ & 36.50 & 0.625 & 0.588 \\
\hline Threshold volume to distension (mL) & 0.405 & 0.071 & 0.179 & $0.267-0.544$ & 41.00 & 0.571 & 0.253 \\
\hline Urge volume to distension (mL) & 0.354 & 0.070 & 0.058 & $0.216-0.492$ & 52.00 & 0.294 & 0.500 \\
\hline Maximal tolerated volume (mL) & 0.481 & 0.136 & 0.857 & $0.214-0.747$ & 102.50 & 0.625 & 0.446 \\
\hline \multicolumn{8}{|l|}{ BBUSQ } \\
\hline Constipation & 0.386 & 0.053 & 0.022 & $0.282-0.490$ & 44.17 & 0.452 & 0.315 \\
\hline Incontinence & $0.717^{\mathrm{a}}$ & 0.047 & 0.000 & $0.625-0.809$ & 39.58 & 0.651 & 0.707 \\
\hline Evacuatory & 0.698 & 0.043 & 0.000 & $0.614-0.782$ & 17.71 & 0.690 & 0.713 \\
\hline Urinary & 0.615 & 0.052 & 0.021 & $0.512-0.718$ & 32.14 & 0.429 & 0.828 \\
\hline WIS & 0.696 & 0.050 & 0.000 & $0.598-0.795$ & 8.50 & 0.649 & 0.693 \\
\hline \multicolumn{8}{|l|}{ SF-36 } \\
\hline Physical function & $0.701^{\mathrm{a}}$ & 0.047 & 0.000 & $0.608-0.794$ & 67.50 & 0.575 & 0.778 \\
\hline Role physical & $0.704^{\mathrm{a}}$ & 0.047 & 0.000 & $0.611-0.797$ & 71.88 & 0.700 & 0.707 \\
\hline Role emotional & 0.673 & 0.051 & 0.001 & $0.574-0.772$ & 79.17 & 0.684 & 0.646 \\
\hline Vitality & 0.644 & 0.053 & 0.005 & $0.540-0.747$ & 53.13 & 0.725 & 0.575 \\
\hline Mental health & $0.730^{\mathrm{a}}$ & 0.043 & 0.000 & $0.645-0.815$ & 67.50 & 0.700 & 0.675 \\
\hline Social function & $0.787^{\mathrm{a}}$ & 0.038 & 0.000 & $0.712-0.862$ & 68.750 & 0.800 & 0.697 \\
\hline Pain & 0.627 & 0.052 & 0.014 & $0.525-0.728$ & 53.130 & 0.500 & 0.787 \\
\hline General health & $0.709^{a}$ & 0.046 & 0.000 & $0.618-0.800$ & 57.500 & 0.632 & 0.743 \\
\hline
\end{tabular}

ROC, receiver operating characteristic; AUC, area under curve; SE, standard error; Cl, confidence interval; ARM, anorectal manometry; BBUSQ, Birmingham Bowel and Urinary Symptoms Questionnaire; WIS, Wexner Incontinence Score; SF-36, Short Form 36, quality of life questionnaire.

aAUC $>0.7$.

al. [8] studied a group of 35 patients referred for investigation of FI. They found no correlation of either ARM or endoanal ultrasound with clinical outcome. A small number of studies aimed to predict the presence or severity of anal incontinence. Pehl et al. [9] undertook a study of 144 healthy volunteers and 559 incontinent patients with varying severities of incontinence (gas, liquid stool, solid stool) and compared motor and sensory functions to severity of incontinence. They found significant differences in the mean RP, mean SP and volume to urge in FI patients, and they showed that overall, ARM had a sensitivity of $91.4 \%$, a specificity of $63.2 \%$ and a diagnostic accuracy of $85.8 \%$ in prediction of the presence of FI. Felt-Bersma et al. [10] demonstrated that the mean $\mathrm{SP}$ was the most discriminatory of all measurements used in the prediction of FI; however, for all measurements, an almost complete overlap with the measurements for patients without incontinence and healthy volunteers was found. Lam et al. [11] under- took a comprehensive study of 600 patients, with the aim of identifying predictive factors for FI. They found that a reduced mean $\mathrm{RP}$ and a reduced mean SP were significantly associated with the presence of FI in both males and females. Additionally, in females, a shorter sphincter length and a lower rectal capacity were associated with FI. In a similar vein, Roos et al. [12] in their study of 159 patients with OASI found no significant difference in functional incontinence scores in women with and without evidenced sphincter defects. They also found no correlation between endoanal ultrasound results and functional incontinence scores. They used ROC analyses to determine cutoff manometric values for the detection of anal sphincter defects and reported that a mean $\mathrm{RP}$ of $95 \mathrm{mmHg}$ and a mean squeeze increment of $53 \mathrm{mmHg}$ would allow $90 \%$ of all sphincter defects to be detected. Reddymasu et al. [13] in their cohort study of 27 patients found that ARM in combination with the use of endoanal ultrasound was 




Fig. 1. Receiver operating characteristic (ROC) curves with areas under the curves values > 0.7: age (A), 5-second squeeze increment (B), Birmingham Bowel and Urinary Symptoms Questionnaire (BBUSQ)-fecal incontinence domain (C), and Short Form 36 (SF-36) social function score (D).

useful in detecting an abnormality in 25 of 27 patients.

The main limitation of this study is that the predominant etiology of FI in this patient population is OASI; however, the proportions seen within this research are representative of the general case mix presenting to a tertiary pelvic-floor service. Additionally, patient involvement and completion of PROMS were entirely voluntary; therefore, a degree of reporting bias may exist as those who completed the PROMs may have been more motivated to do so due to their symptoms being worse and may have had a greater desire to relay this to the clinical team. Additionally, meaningful multivariate logistic regression analyses could not be performed on the data due to the low ratio of positive events to the number of significant factors on the univariate analyses. Another potential limitation was the use of an air-perfused catheter ARM system, which had been in use since the development of the local Pelvic Floor Service. Other more widely accepted ARM systems exist, 
however the air-perfused system has been demonstrated to have comparable performance to water-perfused and solid state systems [14]. Therefore its use throughout this research was continued to allow for consistency in methodology within this patient cohort.

In conclusion, PROMs, which are inexpensive and easily performed, are reliable predictors of maximal treatment in patients with FI and can be readily used both in primary care to aid surgical referrals and in hospital settings, in conjunction with only the sustained SP in ARM, to guide surgical management. Additionally, within our series, BBUSQ assessments of IAS and EAS functions through reporting of symptoms of fecal urgency, inability to defer defecation, and passive FI were found to correlate with ARM evidence of sphincter disruption. Therefore, the absence of these key symptoms may be a useful indicator in determining those who do not need full manometric sphincter assessments. Simple questionnaires assessing the degree of dysfunction and impact on QoL, such as the ones described above, may be useful for general practitioners in the community and specialists in referral centers to allow triage of patients with less severe symptoms who would benefit from conservative treatment in the form of physiotherapy, ultimately reducing the burden on tertiary-level care.

\section{CONFLICT OF INTEREST}

No potential conflict of interest relevant to this article was reported.

\section{REFERENCES}

1. Rao SS; American College of Gastroenterology Practice Parameters Committee. Diagnosis and management of fecal incontinence. American College of Gastroenterology Practice Parameters Committee. Am J Gastroenterol 2004;99:1585-604.

2. Ng KS, Sivakumaran Y, Nassar N, Gladman MA. Fecal incontinence: community prevalence and associated factors--a systematic review. Dis Colon Rectum 2015;58:1194-209.

3. Norton C, Thomas L, Hill J; Guideline Development Group.
Management of faecal incontinence in adults: summary of NICE guidance. BMJ 2007;334:1370-1.

4. Hiller L, Radley S, Mann CH, Radley SC, Begum G, Pretlove SJ, et al. Development and validation of a questionnaire for the assessment of bowel and lower urinary tract symptoms in women. BJOG 2002;109:413-23.

5. Jorge JM, Wexner SD. Etiology and management of fecal incontinence. Dis Colon Rectum 1993;36:77-97.

6. Brazier JE, Harper R, Jones NM, O'Cathain A, Thomas KJ, Usherwood T, et al. Validating the SF-36 health survey questionnaire: new outcome measure for primary care. BMJ 1992;305:160-4.

7. Ruopp MD, Perkins NJ, Whitcomb BW, Schisterman EF. Youden Index and optimal cut-point estimated from observations affected by a lower limit of detection. Biom J 2008;50:419-30.

8. Hill K, Fanning S, Fennerty MB, Faigel DO. Endoanal ultrasound compared to anorectal manometry for the evaluation of fecal incontinence: a study of the effect these tests have on clinical outcome. Dig Dis Sci 2006;51:235-40.

9. Pehl C, Seidl H, Scalercio N, Gundling F, Schmidt T, Schepp W, et al. Accuracy of anorectal manometry in patients with fecal incontinence. Digestion 2012;86:78-85.

10. Felt-Bersma RJ, Klinkenberg-Knol EC, Meuwissen SG. Anorectal function investigations in incontinent and continent patients. Differences and discriminatory value. Dis Colon Rectum 1990;33: 479-85.

11. Lam TJ, Kuik DJ, Felt-Bersma RJ. Anorectal function evaluation and predictive factors for faecal incontinence in 600 patients. Colorectal Dis 2012;14:214-23.

12. Roos AM, Abdool Z, Thakar R, Sultan AH. Predicting anal sphincter defects: the value of clinical examination and manometry. Int Urogynecol J 2012;23:755-63.

13. Reddymasu SC, Singh S, Waheed S, Oropeza-Vail M, McCallum RW, Olyaee M. Comparison of anorectal manometry to endoanal ultrasound in the evaluation of fecal incontinence. Am J Med Sci 2009;337:336-9.

14. Fang JC, Hilden K, Tuteja AK, Peterson KA. Comparison of aircoupled balloon esophageal and anorectal manometry catheters with solid-state esophageal manometry and water-perfused anorectal manometry catheters. Dig Dis Sci 2004;49:1657-63. 